\title{
LESÕES TRAUMÁTICAS dO PARÊNQUIMA PULMONAR: ASPECTOS NA TOMOGRAFIA COMPUTADORIZADA*
}

\author{
Alessandro Severo Alves de Melo ${ }^{1}$, Luiza Beatriz Melo Moreira ${ }^{1}$, Edson Marchiori ${ }^{2}$
}

Resumo As lesões pulmonares são achados freqüentes no trauma torácico, sendo cada vez mais diagnosticadas pela tomografia computadorizada, em especial pelo rápido tempo de aquisição decorrente da técnica helicoidal, que permite a avaliação de pacientes em estado grave, possibilitando a adoção de conduta terapêutica eficiente. Os autores estudaram 150 pacientes vítimas de trauma torácico submetidos a tomografia computadorizada, que apresentaram lesões pulmonares, representadas por contusões, atelectasias, lacerações e hematomas pulmonares. As contusões pulmonares se caracterizaram por consolidações e atenuação em vidro fosco, sendo as lesões pulmonares mais comuns. As atelectasias foram observadas com os padrões subsegmentar e compressiva, e foram a segunda lesão mais comum. As lacerações se apresentaram como consolidações com ar ou nível líquido no interior. Os hematomas pulmonares representaram a lesão pulmonar mais rara, presentes em apenas cinco casos, caracterizados por opacidades arredondadas. Neste trabaIho o trauma torácico fechado predominou, com 120 casos, enquanto o trauma aberto ocorreu em $30 \mathrm{ca}$ sos. As causas de trauma fechado, em ordem decrescente de freqüência, foram: colisão automobilística, atropelamento, queda de altura, acidente de motocicleta e espancamento. A forma penetrante de traumatismo torácico decorreu de duas causas de agressão: lesão por arma de fogo e lesão por arma branca.

Unitermos: Contusão pulmonar; Tomografia computadorizada; Trauma.

Abstract Traumatic lung lesions: computed tomography findings.

Traumatic lesions of the lung are common findings in patients with thoracic trauma. These lesions are increasingly diagnosed using computed tomography, mostly due to the fast acquisition time helical techniques that allow evaluation of critically ill patients and an efficient therapeutic management. The authors studied 150 patients with thoracic trauma submitted to computed tomography that demonstrated lung contusions, atelectasies, lacerations and hematomas. Lung contusions were the most frequent lesions appearing as consolidation or ground-glass attenuation areas. Atelectasies, in subsegmentar and compressive patterns, were the second most common lesions observed. Lacerations appeared as consolidations with air or liquid level. Lung hematomas, characterized by round opacities, were the most rare lung lesions seen in only five cases. In this study, blunt thoracic trauma accounted for the majority of 120 cases whereas penetrating trauma occurred in 30 cases. The causes of blunt trauma in decrescent order of frequency were motor vehicle accidents, pedestrian hit by car, falls, motorcycle accidents and trashing. Penetrating traumas were caused by bullets or knives.

Key words: Lung contusion; Computed tomography; Trauma.

\section{INTRODUÇÃO}

O trauma é uma das principais causas de invalidez e morte no mundo atual, em especial na população mais jovem, sendo que a lesão torácica representa um agravante importante na evolução dos pacientes com trauma multissistêmico, chegando

* Trabalho realizado no Serviço de Radiologia do Hospital Municipal Souza Aguiar, Rio de Janeiro, RJ.

1. Médicos Radiologistas do Hospital Municipal Souza Aguiar, Mestres em Radiologia pela Universidade Federal do Rio de Janeiro (UFRJ).

2. Professor Titular de Radiologia da Universidade Federa Fluminense (UFF), Coordenador Adjunto do Curso de Pós-Graduação em Radiologia da UFRJ.

Endereço para correspondência: Dr. Alessandro Severo Alves de Melo. Rua Cinco de Julho, 273, apto. 1302, Icaraí. Niterói, RJ, 24220-110. E-mail: alessandrosevero@ig.com.br

Recebido para publicação em 26/12/2002. Aceito, após revisão, em 28/1/2003. a determinar $20 \%$ das mortes de origem traumática $^{(1)}$.

O uso da tomografia computadorizada (TC) tem representado um significativo avanço na abordagem moderna das vítimas de trauma. A TC avalia muito bem as lesões traumáticas do crânio e face, da coluna, do tórax, do abdome e da pelve, permitindo um melhor estudo das lesões dos pacientes traumatizados, conseqüentemente desempenhando um papel essencial nos centros hospitalares especializados.

A TC, e especialmente a TC helicoidal, apresentam maior sensibilidade e especificidade que as radiografias de tórax na detecção e avaliação da extensão das lesões traumáticas do parênquima pulmonar, do espaço pleural, da aorta e do diafragma, possibilitando melhor avaliação das lesões e também permitindo o diagnóstico correto de comprometimento extratorácico ${ }^{(2-7)}$.

O trauma pode produzir variadas lesões nos órgãos intratorácicos, sendo as lesões parenquimatosas mais comuns as contusões pulmonares, as atelectasias, as lacerações e os hematomas.

Neste trabalho são discutidas as principais alterações encontradas nas TC de 150 pacientes com lesão pulmonar traumática, sendo estes achados comparados com os da literatura.

\section{CASUÍSTICA E MÉTODO}

Foram reunidos, em estudo prospectivo, 150 casos de lesões traumáticas do 
parênquima pulmonar diagnosticadas no período de janeiro/2001 a agosto/2002, provenientes de quatro instituições do Rio de Janeiro, RJ: Hospital Municipal Souza Aguiar (HMSA), Hospital Barra D'Or (HBD), Hospital Copa D'Or (HCD) e Hospital de Clínicas de Niterói (HCN).

A faixa etária dos pacientes variou de 8 a 82 anos, com média de idade de 35 anos. O sexo masculino predominou, com cerca de 76,7\% $(\mathrm{n}=115)$, sendo apenas 35 pacientes $(23,3 \%)$ do sexo feminino.

Os pacientes foram submetidos a TC do tórax em aparelhos de aquisição helicoidal com $5 \mathrm{~mm}$ de colimação, da fúrcula esternal até o andar superior do abdome, sendo os exames realizados até 12 horas após o trauma torácico.
Neste trabalho os aspectos estudados foram o tipo do trauma torácico (trauma fechado ou aberto), as causas das lesões traumáticas e o padrão das lesões.

\section{RESULTADOS}

As lesões pulmonares foram divididas, segundo a forma de apresentação, em contusões, atelectasias, lacerações e hematomas. As lesões pulmonares foram determinadas por trauma torácico fechado em 120 casos $(80 \%)$ e por trauma torácico aberto ou penetrante em 30 pacientes $(20 \%)$. Em relação ao trauma torácico fechado, houve predomínio de colisão de automóveis, fator causal em 51 casos (34\% do total geral de traumatismos pulmonares), seguida pelo atropelamento, em 41 casos $(27,3 \%)$, queda de altura, em 15 casos (10\%), acidentes de motocicleta, em 9 casos $(6 \%)$, e espancamentos, em 4 casos $(2,7 \%)$. A forma aberta ou penetrante de traumatismo torácico decorreu de duas causas de agressão: a lesão por arma de fogo, presente em 27 casos (18\% do total geral de traumas torácicos), e a lesão por arma branca, observada em 3 casos (2\%).

A contusão pulmonar foi a lesão parenquimatosa mais comum nesta casuística e também o achado isolado mais observado, presente em 145 pacientes $(96,7 \%)$, manifestando-se na TC sob a forma de consolidação (Figura 1), atenuação em vidro fosco, ou uma mescla destes dois padrões (Figuras 2,3 e 4 ).

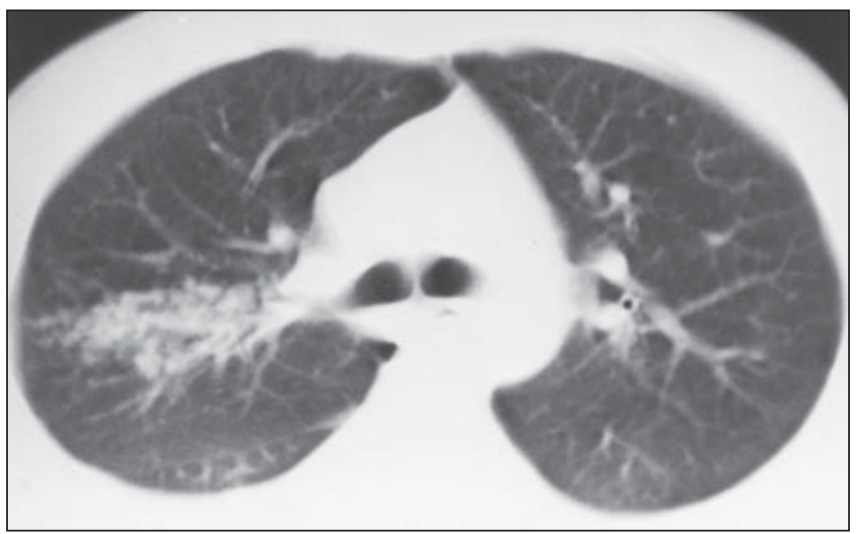

Figura 1. Área de contusão pulmonar caracterizada por consolidação com broncograma aéreo no lobo superior direito.

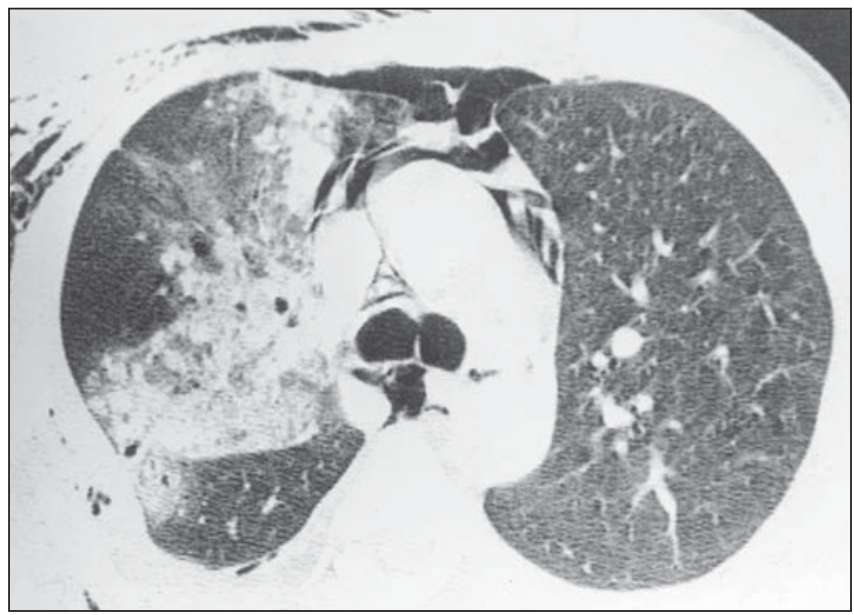

Figura 3. Extensas áreas de consolidação com atenuação em vidro fosco marginal no lobo superior direito, compatíveis com contusão pulmonar. Observamse, ainda, pequeno pneumotórax bilateral, pneumomediastino, enfisema de partes moles no hemitórax direito e derrame pleural deste lado.

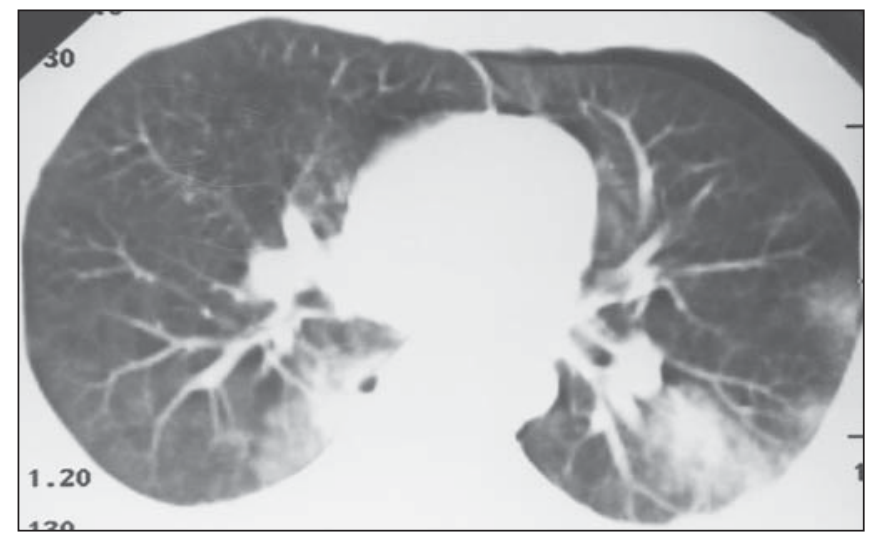

Figura 2. Contusões pulmonares manifestas sob a forma de áreas de consolidação e atenuação em vidro fosco nas regiões posteriores dos lobos inferiores. Há pequena laceração pulmonar no segmento superior do lobo inferior direito e pneumotórax anterior esquerdo.

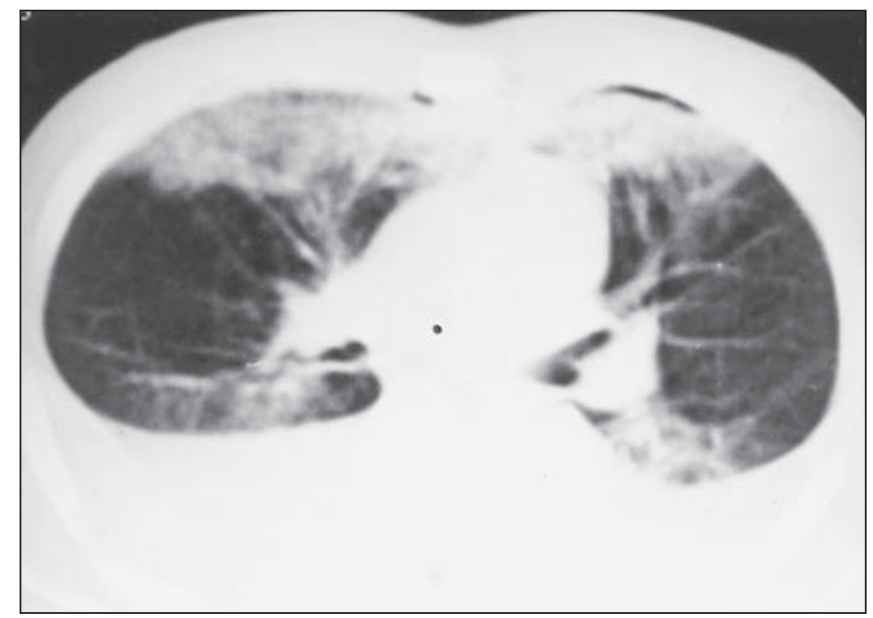

Figura 4. Contusões pulmonares na língula e lobo médio, configurando trajeto linear determinado por projétil de arma de fogo. Há hemotórax bilateral e pequeno pneumotórax esquerdo. 
As atelectasias pulmonares representaram a segunda alteração pulmonar mais comum neste trabalho, sendo observadas em 37 pacientes, correspondendo a 24,7\% dos casos. As formas de atelectasia pulmonar observadas foram dos tipos compressivo e subsegmentar. A atelectasia compressiva foi detectada em 25 casos (Figura 5), e as faixas de atelectasia subsegmentar estiveram presentes em 12 casos.

As lacerações pulmonares foram observadas em 14 casos $(9,3 \%)$, caracterizadas pela presença de ar no interior de uma contusão pulmonar em todos os casos ( $\mathrm{Fi}$ guras 6 e 7), sendo que em 13 pacientes elas decorreram de trauma fechado, e no caso restante, de trauma aberto.

Os hematomas pulmonares constituíram a lesão parenquimatosa menos comum, detectados em $3 \%$ dos casos $(n=5)$,

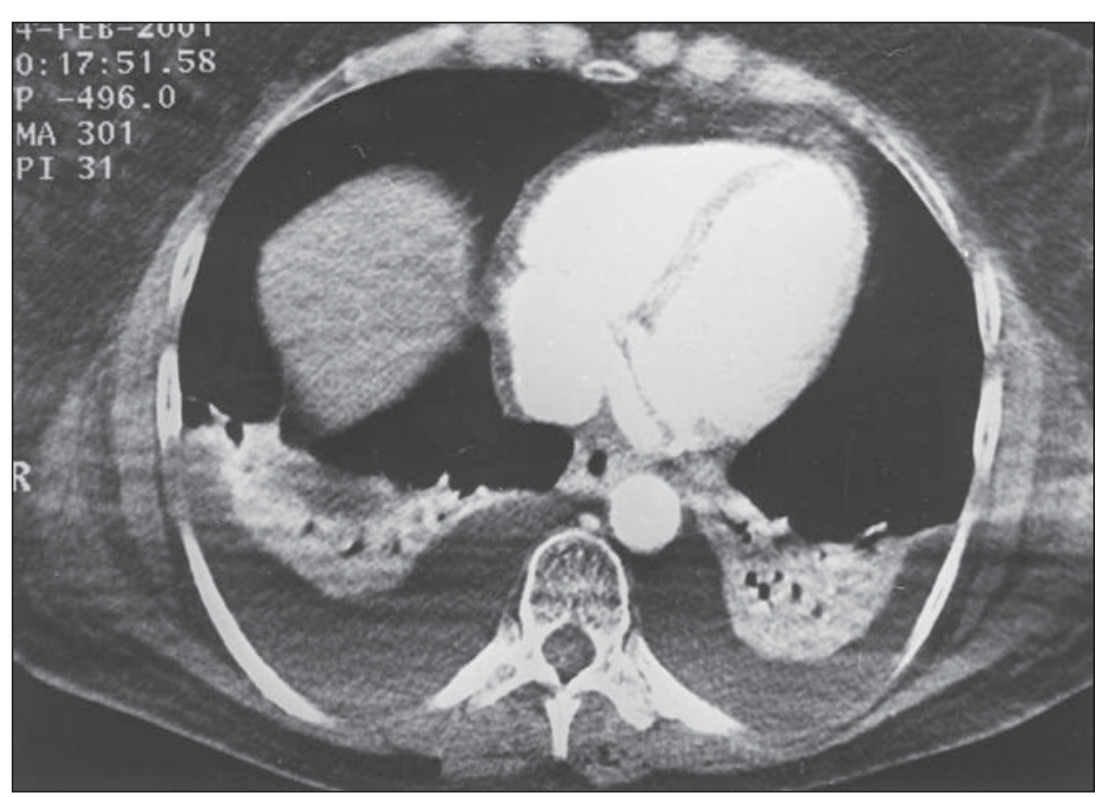

Figura 5. Áreas de atelectasia compressiva nos lobos inferiores, causadas por derrame pleural bilateral.

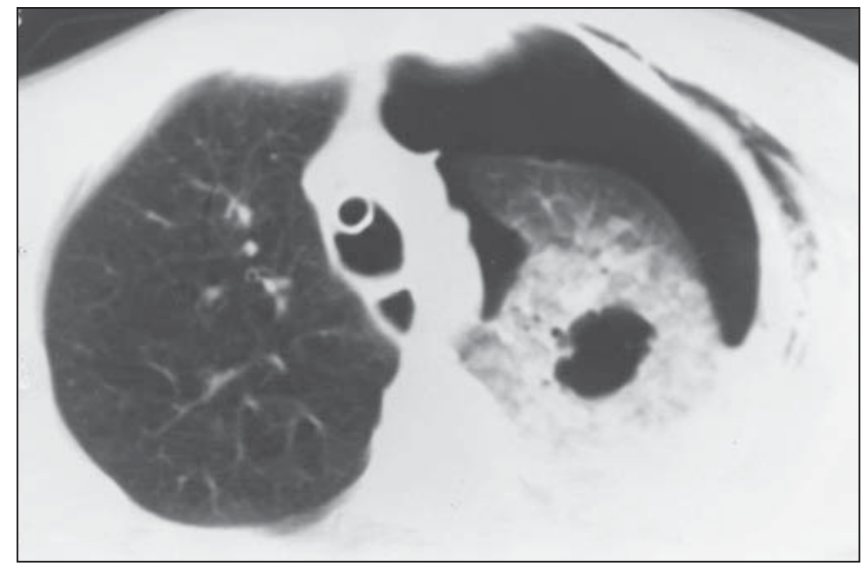

A

Figura 6. Cortes com janela para parênquima pulmonar evidenciando pneumotórax à esquerda, com enfisema de partes moles. Presença de duas áreas de laceração pulmonar, envolvidas por consolidação e atenuação em vidro fosco, que correspondem a contusão pulmonar.

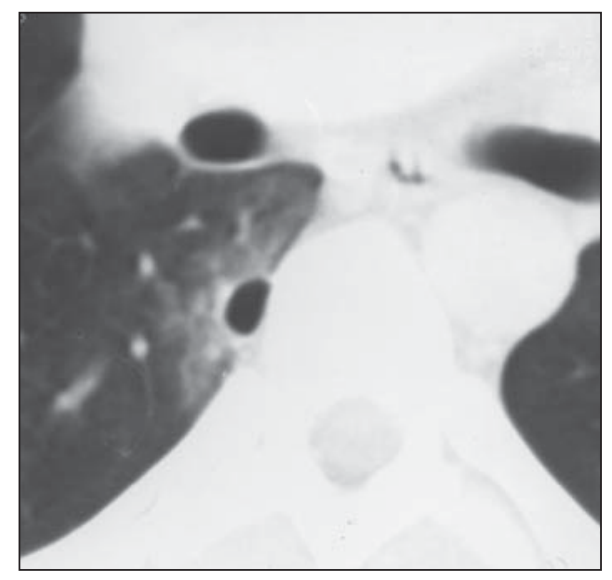

A

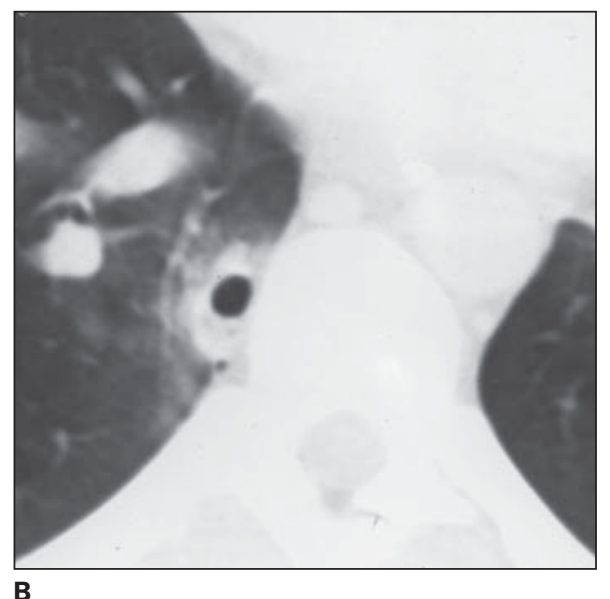

Figura 7. Em A e B, dois níveis diferentes, são vistas duas lacerações pulmonares envoltas por consolidação e atenuação em vidro fosco. Em $\mathbf{C}$, reconstrução no plano sagital mostra o conjunto das lesões.
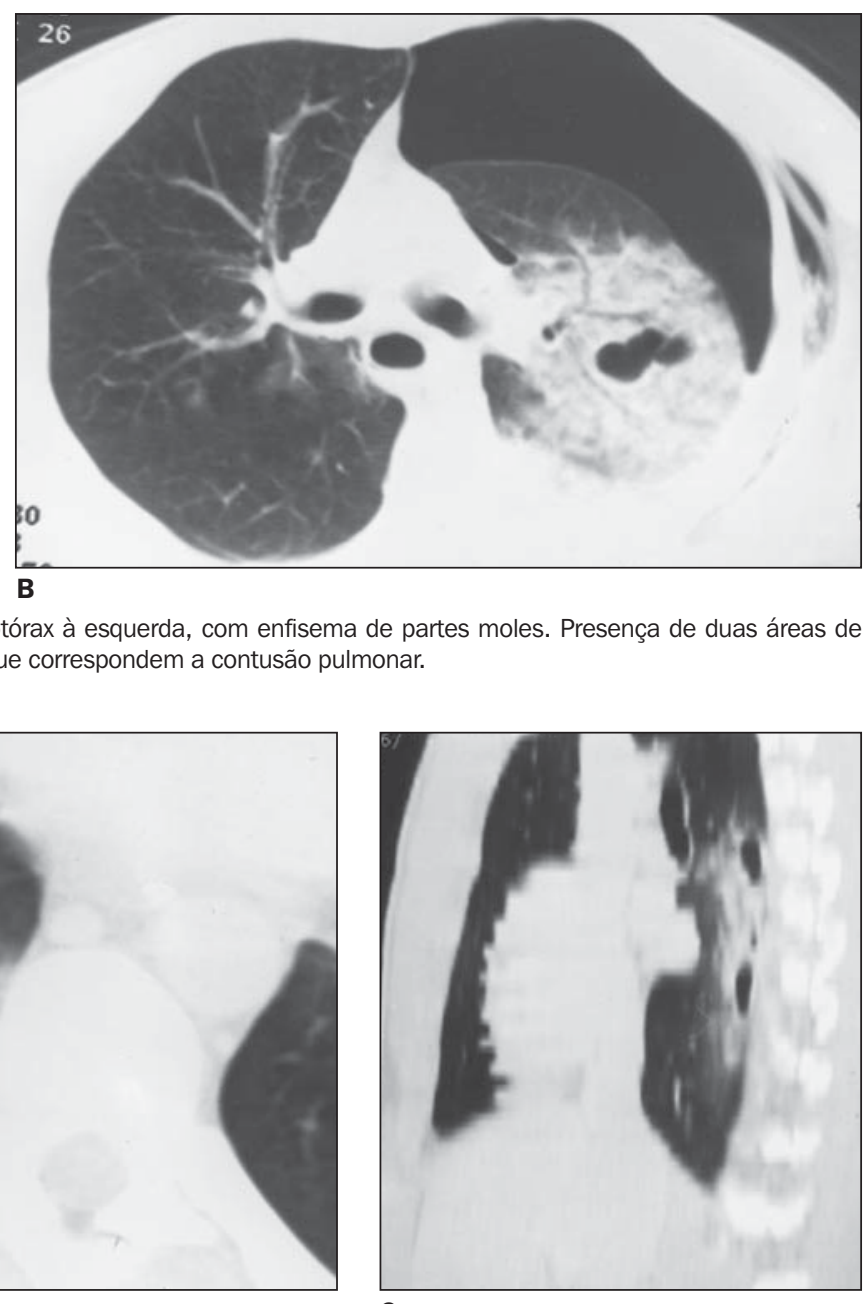

C 
sendo as lesões caracterizadas por opacidades arredondadas, sempre associadas à presença de ar ou nível líquido no interior (Figura 8).

Lesões parenquimatosas foram observadas isoladamente em 40 casos $(26,7 \%)$ e em associação com lesões de outros compartimentos torácicos em 110 casos, equivalendo a $73,3 \%$ dos casos.

Um outro sítio torácico acometido foi o espaço pleural, sob a forma de hemotórax, que ocorreu em 72 casos (48\%), e de pneumotórax, em 61 casos (40,6\%). Enfisema de partes moles e pneumomediastino estiveram presentes em 17 e 15 casos, respectivamente, correspondendo a 11,3\% e $10 \%$. As lesões diafragmáticas foram observadas seis casos (4\%). Hemomediastino e hemopericárdio foram detectados, respectivamente, em cinco e em três casos, correspondendo a 3,3\% e 2\%. Lesão aórtica foi observada em três pacientes, equivalendo a $2 \%$ da casuística.

\section{DISCUSSÃO}

Quando a TC do crânio surgiu, no início dos anos 70, rapidamente se tornou padrão-ouro no diagnóstico das lesões do crânio. Nos anos 80 , foi a vez da TC do abdome ganhar espaço no manejo do trauma abdominal ${ }^{(\mathbf{6})}$. Atualmente, com o advento da TC helicoidal, tem sido avaliado o seu uso no diagnóstico precoce no trauma torácico ${ }^{(\mathbf{1 , 6})}$. O valor da TC no estudo das lesões neurológicas e abdominais já está bem estabelecido, enquanto a utilização deste método para o trauma de tórax, por sua introdução recente, permanece um campo a ser desenvolvido ${ }^{(\mathbf{1 , 6})}$.

A TC do tórax, nos casos de trauma torácico, define mais precisamente a extensão e a gravidade das lesões, que geralmente são subestimadas pelas radiografias convencionais $^{(7,8)}$. Esta observação também é verdadeira no que se refere às lesões pulmonares, como demonstrado por Karaaslan et al. ${ }^{(3)}$, que observaram melhor detecção das contusões pulmonares pela TC, já que as radiografias só foram capazes de mostrar 55\% dessas lesões. McGonigal et $a l .{ }^{(6)}$ encontraram, em pacientes vítimas de trauma torácico, atelectasia em 29 pacientes, sendo somente cinco delas detectadas pelas radiografias. A TC é muito mais sen-

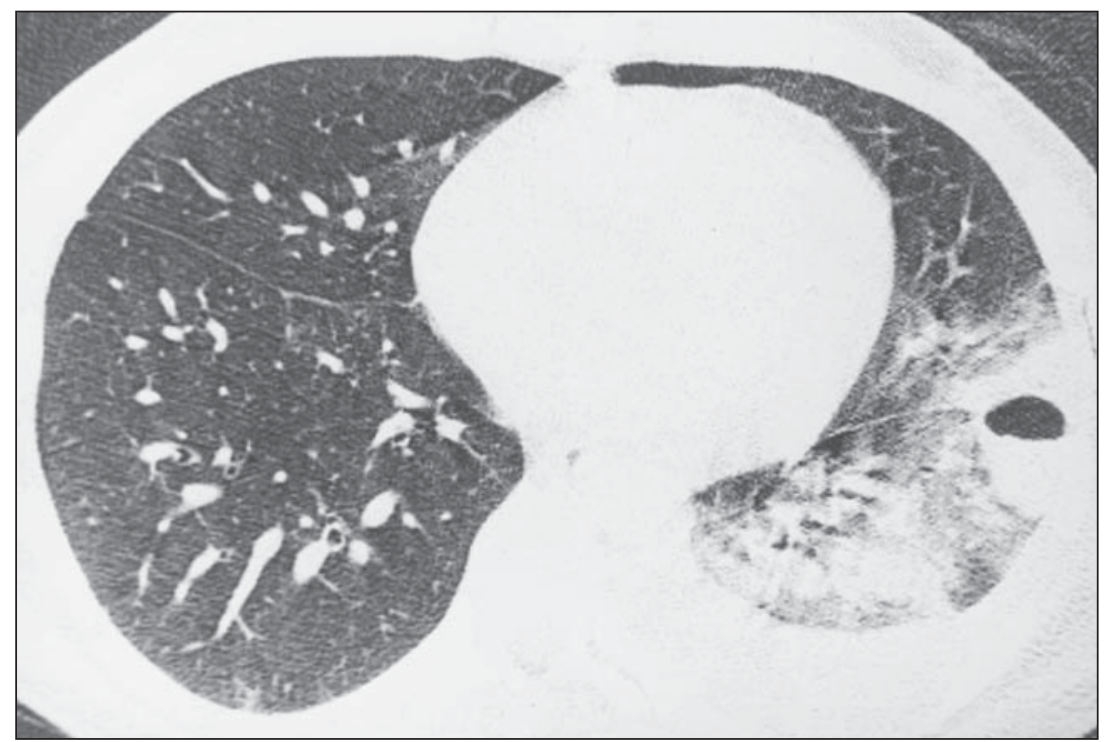

Figura 8. Opacidade nodular, grosseiramente ovalar, com nível líquido, junto à superfície pleural esquerda, que corresponde a hematoma pulmonar. Ao redor da lesão observam-se áreas de consolidação e atenuação em vidro fosco (contusão), além de derrame pleural deste lado.

sível do que a radiografia, inclusive na detecção da laceração pulmonar. No estudo de Wagner e Jamieson ${ }^{(9)}$, feito com 85 pacientes vítimas de trauma torácico, a TC detectou 99 lacerações pulmonares e a radiografia detectou apenas cinco dessas lesões. No nosso estudo observamos uma grande frequiência de lesões pulmonares em pacientes vítimas de trauma, porém não foi realizada avaliação comparativa com as radiografias convencionais.

As lesões pulmonares traumáticas têm como principal causa o acidente automobilístico ${ }^{(\mathbf{8} 9)}$. Marts et al. ${ }^{(\mathbf{8})}$ observaram que os acidentes automobilísticos foram a causa do trauma em 141 casos (83\%), sendo seguidos pelos acidentes de motocicleta, presentes em 11 casos (6\%), pelos atropelamentos, em seis casos (4\%), e por quedas, em quatro casos (2\%). Na nossa casuística houve também predomínio do acidente automobilístico, porém em porcentual menor (34\%); o atropelamento foi a segunda causa mais comum, com $27,3 \%$, seguido por quedas, com $10 \%$, e acidentes de motocicleta, com $6 \%$, notando-se maiores valores porcentuais de atropelamentos e de quedas do que os descritos por Marts et al. ${ }^{(\mathbf{8})}$.

As formas mais freqüentes de lesão pulmonar por nós observadas foram as contusões, as atelectasias, as lacerações e os hematomas.
As contusões pulmonares são definidas como lesões nas quais ocorre dano intersticial e alveolar, com a presença de sangue e edema, sem haver, no entanto, ruptura significativa das paredes alveolares ${ }^{(8-10)}$. As lacerações pulmonares representam lesões parenquimatosas associadas à ruptura das paredes alveolares, sendo, em geral, circundadas por áreas de hemorragia alveolar, e se caracterizam pela presença de ar, líquido ou ambos no interior da lesão(11). As atelectasias representam uma redução volumétrica secundária do pulmão ou de parte dele e se manifestam por opacidades em faixa e condensações em porções pendentes dos pulmões, geralmente associadas a derrame pleural, representando as atelectasias compressivas ${ }^{(\mathbf{3})}$. Os hematomas pulmonares se caracterizam por lesões parenquimatosas de forma arredondada, de conteúdo hemático ${ }^{(4)}$.

As contusões pulmonares são as lesões parenquimatosas traumáticas mais comuns ${ }^{(\mathbf{9 , 1 0})}$. Blostein e Hodgman ${ }^{(\mathbf{1 0})}$ observaram 30 casos de contusão em 76 pacientes $(39,5 \%)$, enquanto atelectasias foram detectadas em 24 casos $(31,6 \%)$ e lacerações foram vistas em nove casos $(11,8 \%)$. Wagner e Jamieson ${ }^{(9)}$ relataram, em 85 pacientes, dados semelhantes, com 143 áreas de contusão pulmonar, 99 lacerações e 16 atelectasias. No nosso estudo também observamos predomínio das contusões pul- 
monares, em 145 pacientes $(96,7 \%)$, enquanto as atelectasias estiveram presentes em 37 casos $(24,7 \%)$ e as lacerações acometeram 14 pacientes $(9,3 \%)$.

$\mathrm{Na}$ TC as contusões aparecem como áreas esparsas, mal definidas, de atenuação em vidro fosco ou de consolidação, que podem apresentar aspecto nodular, ser discretas e até confluentes, normalmente periféricas e não segmentares, podendo ainda se manifestar sob a forma de opacificação difusa de um ou ambos os pulmões ${ }^{(\mathbf{6})}$. Todos estes padrões de lesão pulmonar foram observados no nosso estudo, com aspectos bastante semelhantes aos citados na literatura.

Normalmente, as contusões aparecem dentro de quatro a seis horas depois do trauma e desaparecem em quatro a seis dias, sendo o início abrupto da opacificação parenquimatosa e seu desaparecimento rápido característicos das contusões pulmonares.

As contusões podem aumentar em tamanho e tornar-se mais visíveis em até 48 horas depois do trauma ${ }^{(\mathbf{6})}$. A progressão da contusão após este período, ou demora para absorver por mais de uma semana, indicam erro do diagnóstico inicial ou superposição de outra entidade, como pneumonia, atelectasia, aspiração ou síndrome da angústia respiratória do adulto ${ }^{(6)}$.

A importância clínica da extensão das contusões pulmonares na evolução dos pacientes vítimas de trauma foi avaliada por Wagner e Jamieson ${ }^{(9)}$, que estabeleceram um método quantitativo que divide os lobos pulmonares de acordo com seu volume, demonstrando que os pacientes com contusões ou lacerações pulmonares comprometendo mais de $28 \%$ do tecido pulmonar tiveram indicação de ventilação mecânica. Por outro lado, pacientes que apresentaram menos de $18 \%$ de acometimento do tecido pulmonar não necessitaram de tal procedimento.

Entretanto, a decisão do uso de ventilação mecânica em geral se baseia também nos achados da gasometria, na freqüência respiratória e na presença de choque ou lesões associadas, como trauma crânioencefálico $^{(\mathbf{3 , 5})}$. Blostein e Hodgman ${ }^{(\mathbf{1 0})}$ confirmaram o valor dessa estimativa, correlacionando valores de oxigênio no sangue arterial com o porcentual de volume pulmonar acometido pelas contusões, e concluíram que a taxa de oxigênio reduz com o aumento porcentual de pulmão acometido pelas contusões.

Sivit et al. ${ }^{(11)}$ detectaram trauma torácico significativo em 62 pacientes, vítimas de trauma abdominal fechado, sendo que as alterações parenquimatosas passaram despercebidas pela avaliação radiológica em 34\% dos casos. A TC pode demonstrar, portanto, aspectos sequer suspeitados pela radiografia de tórax, o que se torna relevante, já que, nesse estudo, a mortalidade de crianças sem trauma torácico foi de $1,2 \%$, com trauma torácico unilateral chegou a $10,8 \%$, e com trauma bilateral atingiu $40 \%$, permitindo afirmar que a presença e a gravidade do trauma do tórax pode afetar bastante o prognóstico ${ }^{(11)}$.

As atelectasias representam redução volumétrica secundária do pulmão ou de parte dele e podem ocorrer em vítimas de trauma torácico, sendo caracterizadas por opacidades em faixa (atelectasias subsegmentares) e condensações em porções pendentes dos pulmões, geralmente associadas a hemotórax, que determina compressão do tecido pulmonar adjacente ${ }^{(3)}$.

As atelectasias estiveram presentes em seis casos de Karaaslan et al. ${ }^{(3)}$, sendo quatro subsegmentares (triangulares e periféricas, com predomínio nas bases pulmonares) e duas segmentares, de etiologia compressiva. Estes números são inferiores aos da nossa casuística, em que a atelectasia foi detectada em $24,7 \%$ dos casos ( $\mathrm{n}=$ 37 ), sendo do tipo segmentar em 25 casos e do tipo subsegmentar em 12 casos.

McGonigal et $a l_{.}^{(6)}$ encontraram atelectasia em 29 pacientes, nos exames por TC, sendo somente cinco delas detectadas à radiografia, confirmando a maior sensibilidade da TC também para a demonstração dessas lesões.

As lacerações pulmonares podem resultar de traumas fechados e abertos, tendem a resolver em um período de algumas semanas e, naqueles pacientes em uso de ventilação mecânica, podem aumentar e comunicar-se com o espaço pleural, resultando em pneumotórax ${ }^{(\mathbf{1 2})}$.

As lacerações pulmonares, no nosso estudo, se caracterizaram por consolida- ções, associadas à presença de ar ou nível líquido no seu interior, com os mesmos aspectos de imagem observados na literatura, porém em valores porcentuais não tão altos como os encontrados no estudo de Wagner e Jamieson ${ }^{(9)}$. No nosso estudo encontramos essas lesões em 14 pacientes (9,3\% dos casos de lesão pulmonar), sendo em 13 deles decorrentes de trauma torácico fechado. Wagner e Jamieson ${ }^{(9)}$, em estudo com 85 casos, observaram lacerações em 75 pacientes, correspondendo a $88 \%$ dos casos. As lacerações pulmonares representam as lesões básicas para a formação de hematoma e de cisto pulmonar póstraumático ${ }^{(4)}$.

Os hematomas pulmonares constituem lesões parenquimatosas preenchidas por sangue, podendo ser decorrentes de trauma torácico aberto ou fechado ${ }^{(4)}$, caracterizando-se, nos métodos de imagem, por opacidades arredondadas, com ou sem ar no seu interior ${ }^{(\mathbf{4})}$.

No nosso estudo, que considerou apenas lesões traumáticas torácicas agudas, observamos a presença de hematoma pulmonar em cinco casos, representando apenas $3 \%$ do total. O baixo porcentual de hematomas pulmonares provavelmente decorre do fato de esta lesão ser, em geral, detectada mais tardiamente na evolução dos pacientes, o que não fez parte deste trabalho. Em todos os casos de hematoma pulmonar por nós detectados havia laceração parenquimatosa associada.

A TC representou um significativo avanço no estudo dos pacientes vítimas de trauma, nos mais variados segmentos corporais. No que se refere ao tórax, ela possibilita realizar diagnósticos precisos das lesões intratorácicas, aferir sua gravidade e prognóstico, além de permitir a abordagem terapêutica adequada. $\mathrm{O}$ advento da técnica de aquisição helicoidal veio permitir, em função da sua velocidade, a utilização deste método de imagem em pacientes críticos, instáveis ${ }^{(1-3,6)}$.

O nosso estudo permite concluir que a TC do tórax, nas vítimas de trauma, representa um avanço significativo, por sua capacidade diagnóstica, devendo tornar-se método rotineiro da avaliação do paciente politraumatizado nos centros hospitalares que possuem aparelhos helicoidais. 


\section{REFERÊNCIAS}

1. Groskin SA. Selected topics in chest trauma. Radiology 1992;183:605-17.

2. Dyer DS, Moore EE, Mestek MF, et al. Can chest CT be used to exclude aortic injury? Radiology 1999;213:195-202.

3. Karaaslan T, Meuli R, Androux R, Duvoisin B, Hessler C, Schnyder P. Traumatic chest lesions in patients with severe head trauma: a comparative study with computed tomography and conventional chest roentgenograms. J Trauma 1995;39:1081-6.

4. Marchiori E, Moreira DM. Lesões traumáticas do parênquima pulmonar. Considerações radiológicas sobre cinco casos. Radiol Bras 1984;17:164-71.

5. LoCícero J III, Mattox KL. Epidemiology of chest trauma. Surg Clin North Am 1989;69:15-9.

6. McGonigal MD, Schwab CW, Kauder DR, Miller WT, Grumbach K. Supplemental emergent chest computed tomography in the management of blunt torso trauma. J Trauma 1990;30;1431-4.

7. Feliciano DV. Patterns of injury. In: Feliciano DV, Moore EE, Mattox KL, eds. Trauma. 3rd ed. Stanford: Appleton \& Lange, 1996:85-103.

8. Marts B, Durham R, Shapiro M, et al. Computed tomography in the diagnosis of blunt thoracic in- jury. Am J Surg 1994;168:688-92.

9. Wagner RB, Jamieson PM. Pulmonary contusion. Evaluation and classification by computed tomography. Surg Clin North Am 1989;69:31-40.

10. Blostein PA, Hodgman CG. Computed tomography of the chest in blunt thoracic trauma: results of a prospective study. J Trauma 1997;43:13-8.

11. Sivit CJ, Taylor JA, Eichelberger MR. Chest injury in children with blunt abdominal trauma: evaluation with CT. Radiology 1989;171:815-8.

12. Wagner RB, Crawford WO Jr, Schimpf PP. Classification of parenchymal injuries of the lung. Radiology 1988;167:77-82. 